\title{
The Performance of Structure-Controller Coupled Systems Analysis Using Probabilistic Evaluation and Identification Model Approach
}

\author{
Mosbeh R. Kaloop, ${ }^{1,2,3}$ Jong Wan Hu, ${ }^{1,2}$ and Yasser Bigdeli ${ }^{4}$ \\ ${ }^{1}$ Department of Civil and Environmental Engineering, Incheon National University, Incheon, Republic of Korea \\ ${ }^{2}$ Incheon Disaster Prevention Research Center, Incheon National University, Incheon, Republic of Korea \\ ${ }^{3}$ Public Works and Civil Engineering Department, Mansoura University, Mansoura 35516, Egypt \\ ${ }^{4}$ Department of Civil and Environmental Engineering, Louisiana State University, Baton Rouge, LA, USA
}

Correspondence should be addressed to Jong Wan Hu; jongp24@incheon.ac.kr

Received 18 March 2017; Accepted 9 May 2017; Published 8 June 2017

Academic Editor: Sang-Youl Lee

Copyright ( 2017 Mosbeh R. Kaloop et al. This is an open access article distributed under the Creative Commons Attribution License, which permits unrestricted use, distribution, and reproduction in any medium, provided the original work is properly cited.

\begin{abstract}
This study evaluates the performance of passively controlled steel frame building under dynamic loads using time series analysis. A novel application is utilized for the time and frequency domains evaluation to analyze the behavior of controlling systems. In addition, the autoregressive moving average (ARMA) neural networks are employed to identify the performance of the controller system. Three passive vibration control devices are utilized in this study, namely, tuned mass damper (TMD), tuned liquid damper (TLD), and tuned liquid column damper (TLCD). The results show that the TMD control system is a more reliable controller than TLD and TLCD systems in terms of vibration mitigation. The probabilistic evaluation and identification model showed that the probability analysis and ARMA neural network model are suitable to evaluate and predict the response of coupled buildingcontroller systems.
\end{abstract}

\section{Introduction}

In order to maintain structures safety and service, the application of controllers has been an important option for skyscrapers and important structure all over the world. Controlling systems are designed to protect buildings; bridges; and industrial plants from severe vibrations in seismically active regions [1]. There are different controlling systems, also known as earthquake protective systems, such as simple passive devices and fully active and hybrid systems [1-6].

Passive control systems are being progressively used in vibration control of structures subjected to dynamic loads [7]. The most commonly used passive control systems are tuned mass damper (TMD), tuned liquid damper (TLD), and tuned liquid column damper (TLCD). Tuned liquid column damper, proposed by Sakai et al. [8], combines the liquid mass and orifice damping effect to minimize the vibration. The TLD also uses similar concept to reduce the structural vibration. The advantages and disadvantages of using each passive control system for vibration mitigation are presented in $[1,3,7,9]$. Chen et al. [10] presented a passive control system for a high-rise building in Beijing. They found that the passive control is an economic system that can be used to decrease the displacement response of structures under seismic or dynamic loads. In addition, Kasai et al. [11] concluded that the most common passive control systems are used in Japan and they found that the performance of passive control dampers is higher with earthquake effects. Bigdeli and Kim [3] and Bigdeli [9] have applied different cases of simulation and experimental studies for different cases of passive control systems. They applied the TMD, TLD, and TLCD controller systems and found that the TMD performance system is better to reduce the vibration of structures in frequency domain. Moreover, many papers are published on the performance investigation of these controlling systems in time and frequency domains [3, 9-15]. 
This study applies a novel analysis in time and frequency domains based on probabilistic and identification theories.

The time series analysis was used widely to evaluate the performance and identify the damage of structures in real monitoring measurements and experimental studies [15-20]. Kaloop and Hu [17] and Kaloop and Kim [18] have used the statistical analysis to evaluate the performance of bridges under moving loads and damage effects. The traditional probabilistic evaluation was used to assess and evaluate the seismic effects and risks due to earthquakes [21-23]. It, also, can be used to evaluate structures lifespan [24]. In addition, the probabilistic evaluation method was used as an additional procedure to assess the behavior of structures under seismic effects; moreover, the behavior and damage level of structures were expressed using the probability of exceeding the limit state by site-specific earthquakes during design service life of structures [21]. Kralik [25] utilized the probability analysis to estimate the containment failure of concrete structures. Matos et al. [26] evaluated the behavior of simple beam experimentally and numerically using a linear and nonlinear probability analyses and concluded that the nonlinear probability analysis can be used to estimate the behavior of structures.

Performance models identification was used to predict the behavior of structures under dynamic loads. The predicting models were designed based on input and output or output only of the input and response performance of structures $[27,28]$. This study is focused on input-output models based on experimental studies. The artificial neural networks (ANN) are one of the most accurate and widely used predicting models that have fruitful applications in many engineering problems $[28,29]$. The basics of ANN are presented in $[28,29]$; in addition, the application of ANN to predict the performance and damage detection of structures showed that the ANN is a good tool that can be used in this area [18, 19]. Bigdeli and Kim [12] used ANN to predict the behavior of irregular buildingcontroller system under dynamic loads and found that the ANN can be used to identify the performance of building structures. In addition, Li et al. [30] found that the ANN is feasible to model the linear and nonlinear performance of structures under seismic loads. Autoregressive models gave the best performance results when coupled with ANN [28]. Khashei and Bijari [27] introduced an integrated ANN with autoregressive integrated moving average (ARIMA) to identify the time series problems and found that it is more accurate than ANN. However, in this study, the autoregressive and autoregressive moving average are utilized and evaluated with ANN to develop a prediction model that can be used to identify the behavior of structures controllers under dynamic loads.

The main objective of this study is to investigate the probability analysis and prediction models to evaluate the behavior of an experimental study for a three-story building attached with TMD, TLD, and TLCD controlling systems. Also, a novel application for the probability in time and frequency domains is evaluated and discussed for the controlling systems. Furthermore, an autoregressive moving average-neural network model identification response is designed to predict and investigate the behavior of structures under dynamic loads.

\section{Materials and Methods}

2.1. Response Theory and Experimental Setup. The mechanical configuration of a controlling system consists of a spring and a mass attached on the top of a multistory structure. This system is used as a vibration absorber in order to mitigate structural vibrations. The dominant general equation of motion used for the coupled structure and the absorber for a three-dimensional (3D) structure is given as follows [3, 31]

$$
M \ddot{x}+C \dot{x}+K x=-M r_{g} \ddot{x}_{g},
$$

where $M, C$, and $K$ denote mass, damping, and stiffness matrices of the structure, respectively. The $\ddot{x}, \dot{x}$, and $x$ represent the acceleration, velocity, and displacement response of the structure, respectively. The excitation signal is indicated by $x$ and $r_{g}$ which are defined as the displacement and the ground influence vectors, respectively.

A scaled structural model of a 3D 3-story momentresisting steel frame building structure is selected to conduct the experiments; see Figure 1(a). This study evaluates a steel structure system, widely used in high-risk areas $[6,9]$. The characteristics of the structure are as follows: the total height is $930 \mathrm{~mm}$ and the natural frequency is $2.7 \mathrm{~Hz}$. The diameter of the cross-section area of every column is $5 \mathrm{~mm}$ and the story height equals $310 \mathrm{~mm}$. All degrees of freedom (DOFs) are restricted at the base level. Each floor configuration includes a rigid steel square plate of $300 \mathrm{~mm} \times 300 \mathrm{~mm}$ which weights 3,436 gr with three DOFs for translations in $x$ - and $y$ directions as well as a rotation around a vertical line passing through the center of mass at every floor. The total number of DOFs (42) after application of the boundary conditions (i.e., rigid diaphragm, Guyan reduction of vertical DOFs, and rotational DOFs around $x$ - and $y$-axes) equals 9. The input signal generated artificially through shaking table [29] generates an acceleration signal having a waveform in which parameters such as strong motion duration, ramp times, and number of strong motion peaks are variables. The input and output signals generation and measurements are presented in $[3,31-33]$.

Three passive controllers included in this study, TLD, TLCD, and TMD systems, were used as shown in Figures 1(b), 1(c), and 1(d), respectively. TLCD and TLD devices are fabricated by using a light fiberglass material, while the TMD system is fabricated by using steel material. The total weight of each device is less than $3 \%$ of the whole steel structure. The controllers' weight was kept constant during the conduction of the experiments for all systems. The water inside the containers was in a direct contact with the atmosphere and it also could freely move inside the container. In order to compare the results properly, the following points are considered: firstly, all the three devices have the same primary mass, which means that TLCD and TLD and TMD are identical in weight. Secondly, the same amount of solid or water mass was added to the system including water mass to TLCD and TLD and solid mass to TMD at each step of the tests. Moreover, the total mass used in this study is $1.80 \mathrm{Kg}$. 


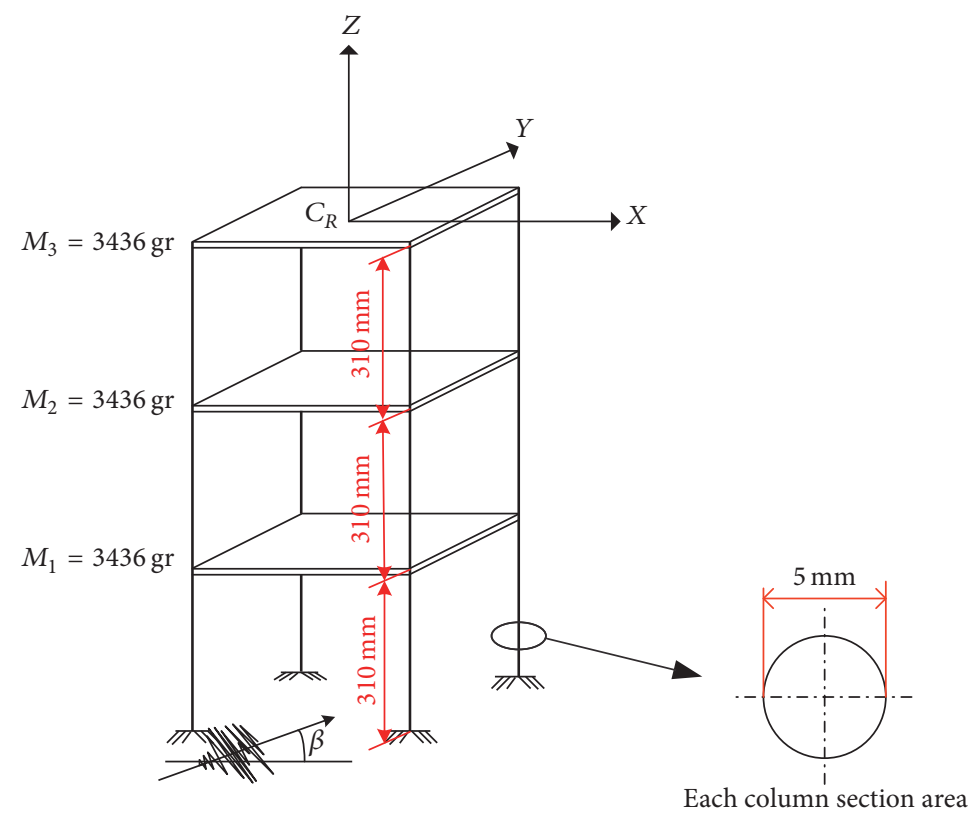

(a)

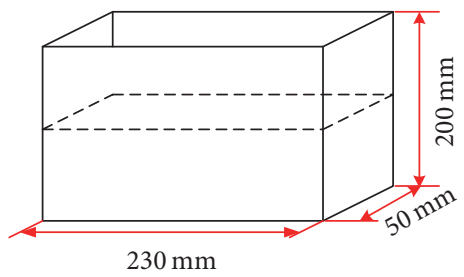

(b)

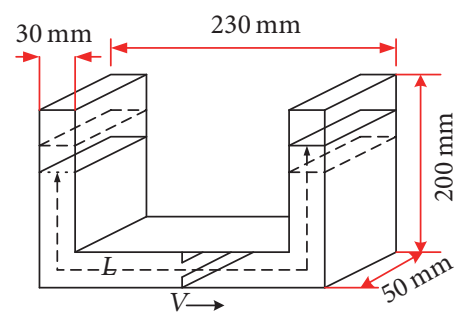

(c)

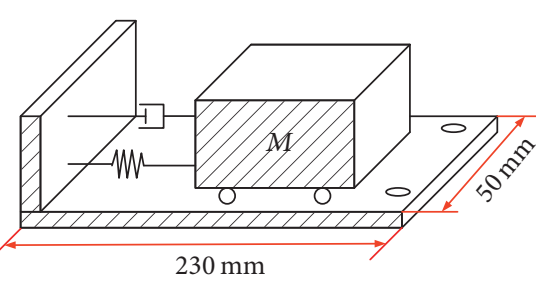

(d)

FIGURE 1: Experimental instruments and setup process, (a) building model, (b) TLD system, (c) TLCD system, and (d) TMD system.

Lastly, the dimensions of the devices are designed in a way to be identical in order to ensure the minimum effects on results.

Figure 2 represents the experimental setup for the controlling system. As shown in Figure 2, a regular structure system is used and the accelerometer sensor is used to measure the response of the three-floor roofs. In this study, the maximum response for the third floor is evaluated.

2.2. Statistical Analysis. The statistical analysis for the controlling system is applied in two ways; conventional (Mean, Skewness, and Kurtosis) and advanced (probability analysis). The statistical moments are used previously to assess the behavior of structures and evaluate structures' monitoring systems $[17,18]$, and also the probability analysis is used to analyze the monitoring system of structures, wind energy, and aerospace evaluation [17, 20, 34, 35]. In this study, the two statistical methods are used to evaluate and assess the behavior of the controlling systems. It is noteworthy that no studies have implemented the probability analysis in control system analysis in time and frequency domain.

In time domain, the Weibull distribution (WD) analysis is widely used to evaluate the behavior of structures [20]. The WD is a probability distribution used to measure the reliability of structures due to its versatility and relative simplicity [36-38], while the reliability of structures can be evaluated by analyzing the interaction between two probability distributions [39]. In WD analysis, the shape $(k)$ and scale $(c)$ parameters are used to extract the Weibull probability density function (PDF) and cumulative distribution function (CDF) $[20,34]$. These parameters can determine the optimum performance of the behavior of structure-controller system $[20,40]$ :

$$
f(m)=\left(\frac{k}{c}\right)\left(\frac{m}{c}\right)^{k-1} e^{-(m / c)^{k}},
$$

for PDF distribution,

$$
F(m)=1-e^{-(m / c)^{k}}, \quad \text { for CDF distribution, }
$$

where $m$ is the time series of the structure behavior measurements (acceleration or displacement), $k>0$ is the dimensionless shape parameter, and $c>0$ is the scale parameter in the measurement unites. Several methods are used to estimate the WD parameters [40]. In this study, the least square method is applied as it is more efficient than other methods [40]. The estimation of the WD parameters in (2) 


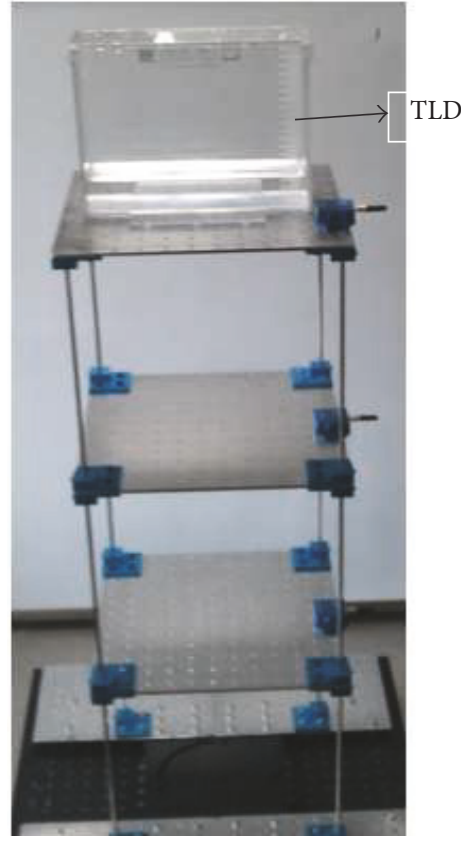

(a)

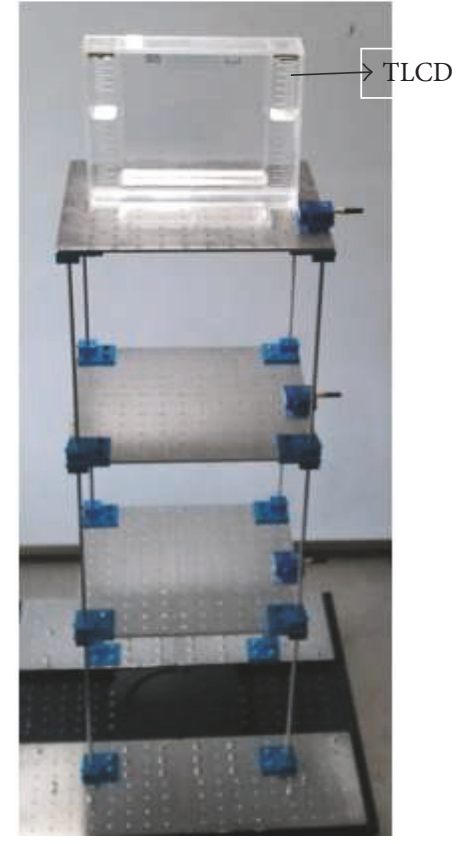

(b)

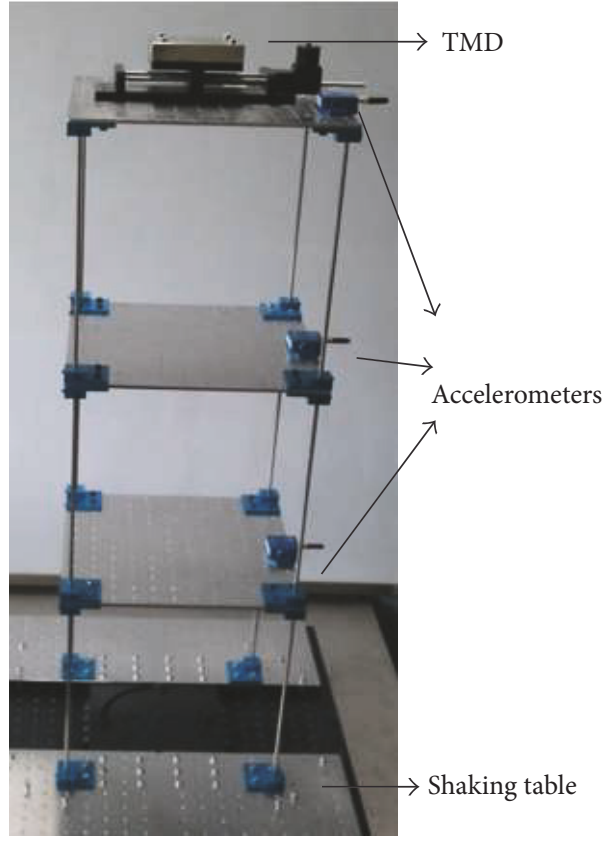

(c)

FIGURE 2: The experimental design for the structure-controller system, (a) TLD, (b) TLCD, and (c) TMD.

refers to probability changes of the response of structures [36]. For instance, the $k$ value measured the failure of the structure response [20]. The smallest value of $k$ parameters refers to more stability and long life [20,36, 41]. In addition, the parameters changes refer to the changes of the peak of PDF curve distribution; it means that the structure behavior should be changed [20, 36-38].

In frequency domain, Trauth [42] presented the LombScargle (LS) periodogram considered a modify power spectrum with an exponential theoretical probability distribution with mean unit, and it is used to estimate the power spectrum density of the measurements signals $[39,42]$. In this study, the LS normalized periodogram $\left(P_{a c}(\omega), \omega=2 \pi f\right)$ is calculated using the time series acceleration measurements for each control system based on least square fit of harmonic function of acceleration data [42]. Therefore, the probability of extracted $P_{a c}(\omega)$ can be found in between some positive quantities $Z$ and $Z+d z$ being $\exp (-Z) d z$ [42]. With assuming the $M$ independent frequency contents, the probability of none of them having a larger value than $Z$ is $(1-\exp (-Z))^{M}[17,42]$. To compute the false-alarm probability of the null hypothesis, the insignificant probability for the peak of the periodogram can be extracted by [42]

$$
P(>Z)=1-\left(1-e^{-Z}\right)^{M} .
$$

Herein, to estimate the significant frequency, two steps are processed; first: the LS periodogram is used to normalize the signal frequency; then the significance level of any peak in the power spectrum can be calculated [42]. The probability calculation in (3) indicates the false-alarm probability of the null hypothesis. Therefore, a low probability indicates a high significant power spectrum peak. However, the false-alarm probability peaks are referred to high significance of the structures' dynamic behavior [17].

2.3. Model Identification Approach. In this study, the proposed model is built based on feedforward neural network model with one hidden layer to provide the third-floor behavior, as presented in Figure 3. The parametric identification models are used to detect the behavior of structures [43-45]. The autoregressive (AR) and autoregressive moving average (ARMA) are among the common and simplified parametric models $[28,43]$. In this study, the two methods are evaluated with feedforward neural network solution. The components of the proposed model are described and explained with details in [43]. A multi-input single-output (MISO) three-layer model is designed for building behavior analysis (Figure 3). The input parameters of the model are the time series of the seismic signal (input shaking table load) and response of structure measurements. The hyperbolic tangent $(f(\cdot))$ and linear activation $(F(\cdot))$ functions are used for the hidden and output layers, respectively. This model is designed to detect the nonlinearity of the structure behavior $[28,44]$. The ARMA neural network (ARMANN) process is a time series consisting of a random error component and a nonlinear combination of inputs values and past residuals [43]. The ARNN model is a special case of the ARMANN model. However, in general the ARMANN process can be summarized in the following equations [43]. 


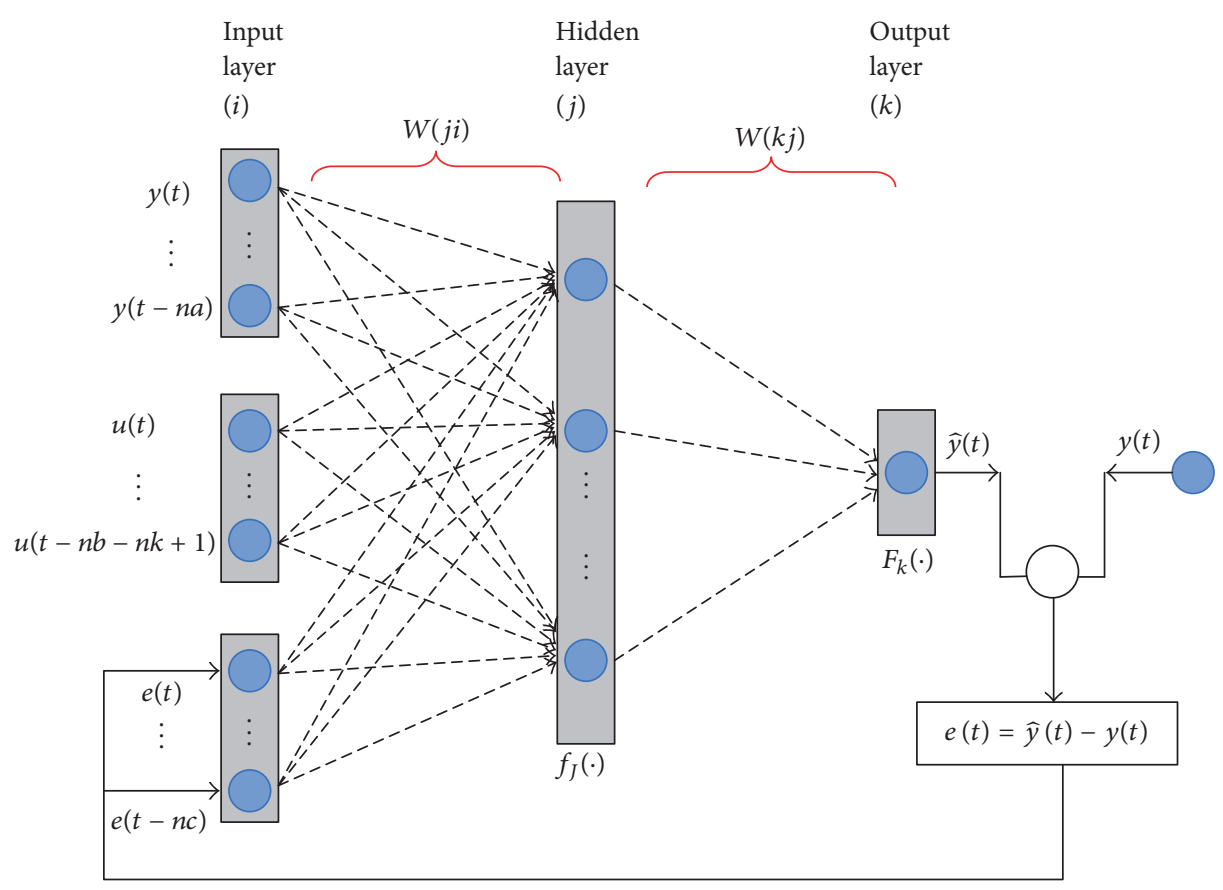

FIGURE 3: Autoregressive Average Moving-Neural Network (ARMANN) structure.

The regression vector is

$$
\varphi(t)=\left[y(t) \cdots y\left(t-n_{a}\right) u\left(t-n_{k}\right) \cdots u\left(t-n_{b}-n_{k}+1\right) e(t-1) \cdots e\left(t-n_{c}\right)\right]^{T}
$$

and prediction is

$$
\widehat{y}\left(\frac{t}{\theta}\right)=g(\varphi(t), \theta),
$$

where $\varphi(t)$ is a vector containing the input dynamic load $(u)$, output behavior $(y)$, and model errors $(e), \theta$ is a vector containing the weights, and $g$ is the function realized by NN. $\hat{y}$ is the prediction vector, $n_{a}$ is the past output used for determining the prediction, $n_{b}$ is the past input, $n_{k}$ is the time delay, and $n_{c}$ is the past residuals.

The relationship between the output and inputs has the following mathematical representation [43]:

$$
\begin{aligned}
& \widehat{y}(t)=F_{k}\left(\sum_{j=0}^{q} W_{k j} f_{j}\left(\sum_{i=0}^{m} W_{j i} Z_{i}+\sum_{l=0}^{p} W_{j l} e(t-l)+w_{j 0}\right)\right. \\
& \left.+w_{k 0}\right)
\end{aligned}
$$

where $W_{k j}, W_{j i}$, and $W_{j l}$ are representing the model parameters often called connection weights; $l$ refers to the input nodes of the model errors only; $m$ is the number of autoregressive (AR) input nodes; $p$ is the number of moving average (MA) parameters input nodes; and $q$ is the number of hidden nodes; $w_{j 0}$ and $w_{k 0}$ are the bias parameters; $Z_{i}$ refers to the AR inputs parameters $(y(t) ; u(t))$.

The main purpose of this model is to determine a mapping from the selected input data to the set of possible weights. So that the network will produce predictions vector, which in some sense are close to the true output vector. The determination of the optimal weight values may be completed by using a trial and error process on an error function (see $(7)$ ), which is called loss function or mean square error $(E)$ of the training process. The loss function is a criterion defined in the mathematical approach as below ( $n$ is the number of observations):

$$
E=\frac{1}{n} \sum_{t=1}^{n}(y(t)-\hat{y}(t))^{2}
$$

The presented strategy tries to minimize the loss function through the model design. By following so, the provided output becomes closer to the controller response measurements (optimum outputs). Eventually, the optimum outputs are going to be predicted after having the input data received through enough number of trial and error efforts. Considering the desired output as a control signal, the network will be known as a controller. In addition, to assess the performance of the model, the mean absolute error $(M)$ is determined with 


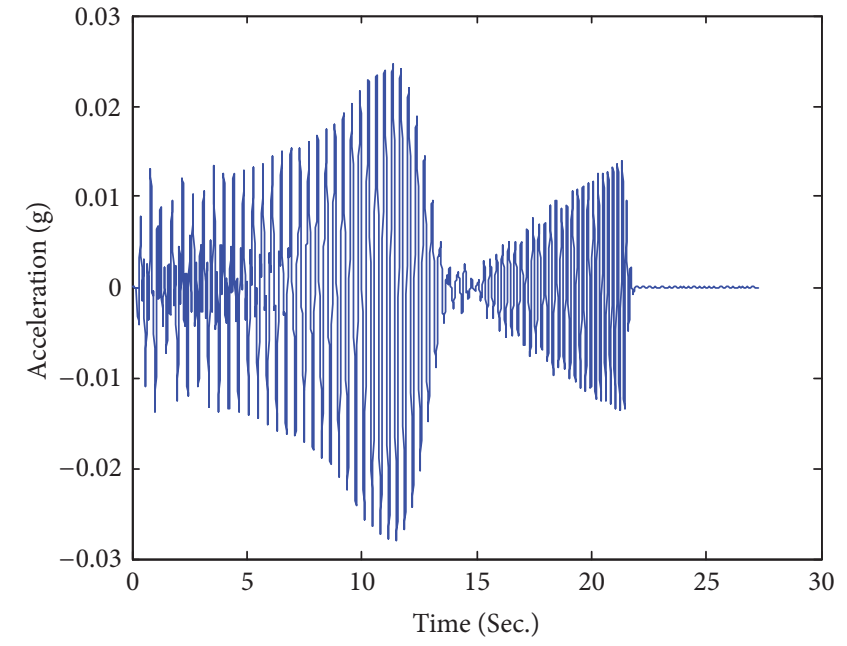

(a)

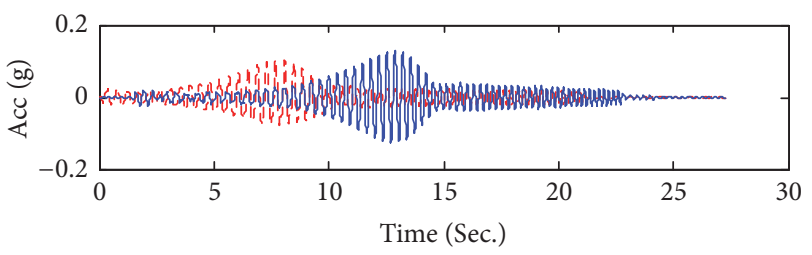

--- TMD

_ Uncontrolled

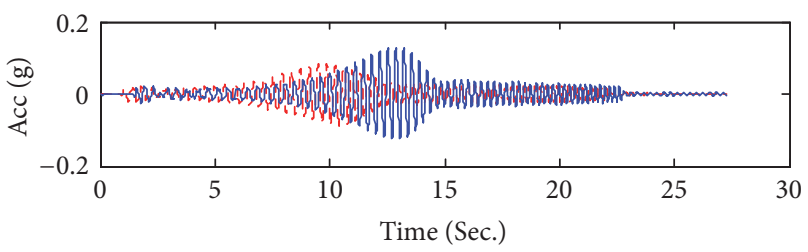

--- TLD

— Uncontrolled

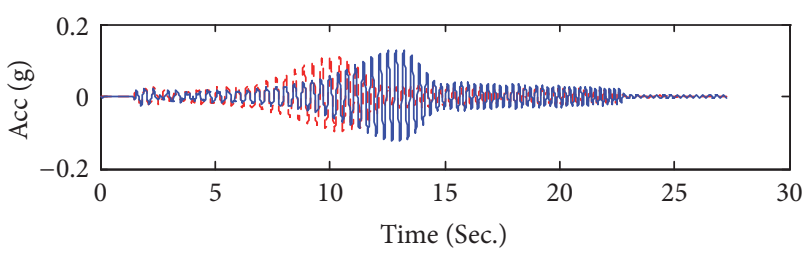

- - TLCD

_ Uncontrolled

FIgURE 4: (a) The excitation signal of seismic design and (b) acceleration responses for the top floor of the uncontrolled structural, TMD, TLD, and TLCD controlled system.

TABLE 1: Statistical parameters for the acceleration and displacement response.

\begin{tabular}{lcccccccc}
\hline \multirow{2}{*}{ Statistical parameter } & \multicolumn{2}{c}{ Uncontrolled } & \multicolumn{2}{c}{ TMD } & \multicolumn{2}{c}{ TLD } & \multicolumn{2}{c}{ TLCD } \\
& Acc $(\mathrm{g})$ & Disp $(\mathrm{mm})$ & Acc $(\mathrm{g})$ & Disp $(\mathrm{mm})$ & Acc $(\mathrm{g})$ & Disp $(\mathrm{mm})$ & Acc $(\mathrm{g})$ & Disp (mm) \\
\hline Mean & 0.022 & 0.611 & 0.017 & 0.558 & 0.016 & 0.507 & 0.018 & 0.573 \\
Maximum & 0.13 & 3.862 & 0.10 & 3.420 & 0.08 & 2.708 & 0.11 & 3.598 \\
Kurtosis & 7.20 & 7.16 & 7.20 & 6.24 & 5.71 & 5.31 & 7.32 & 6.39 \\
Skewness & 2.04 & 2.11 & 1.91 & 1.94 & 1.61 & 1.71 & 1.99 \\
\hline
\end{tabular}

$E$ to measure predicting performance of the proposed model in comparison to other predicting models.

\section{Results and Discussions}

3.1. Response Statistical Analysis. The time series analyses in time and frequency domains are presented in this section. Figure 4 illustrates the time series of seismic shaking table signals and acceleration responses for the uncontrolled system and also for the controlled systems by using TMD, TLD, and TLCD where the entire system was under seismic load effects.

From Figure 4, the maximum seismic excitation occurred at 11.53 seconds, while the maximum acceleration response of the uncontrolled, TMD, TLD, and TLCD systems occurred at $12.71,7.85,10.40$, and 10.95 seconds, respectively. The
TMD structure response is delayed by 4.86 seconds, and it means that the TMD control system is more sensitive to the loads than TLD and TLCD systems. In addition, the peak displacement reductions are significant with 16.9, 15.0, and $16.0 \%$ for the TMD, TLD, and TLCD controlling systems, respectively. Table 1 reports the moments of statistical analyses for the acceleration and computed displacements of the three models. The mean, maximum, Skewness, and Kurtosis are often calculated in order to examine the raw time series data obtained for the excitations and responses of a structure. Herein, the statistical analysis is performed for the absolute measurements acceleration and calculations of displacements.

The acceleration absolute mean shows that the structure vibration is decreased by $22.7,27.3$, and $18.2 \%$ with TMD, TLD, and TLCD, respectively. The maximum acceleration 


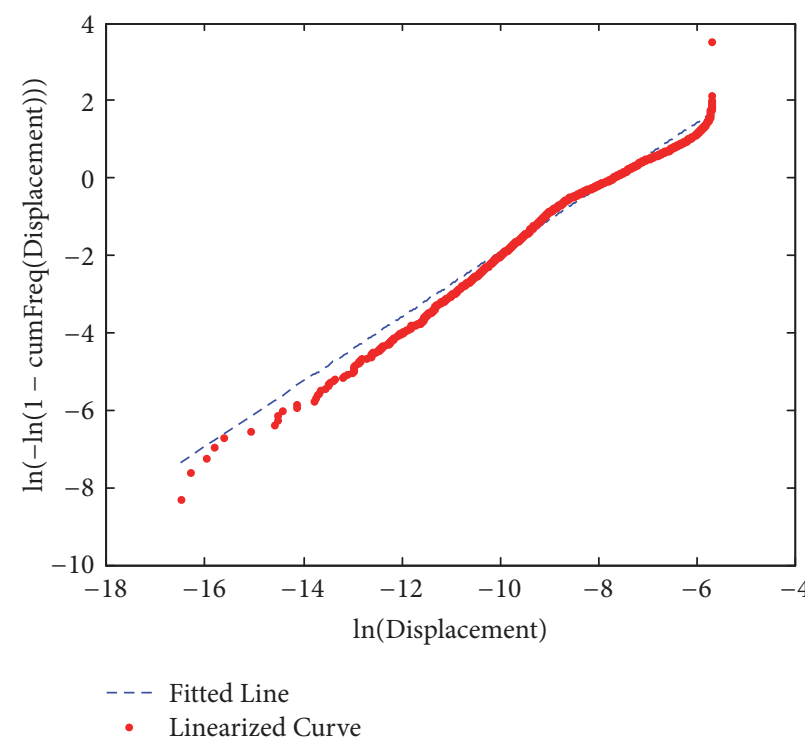

(a)

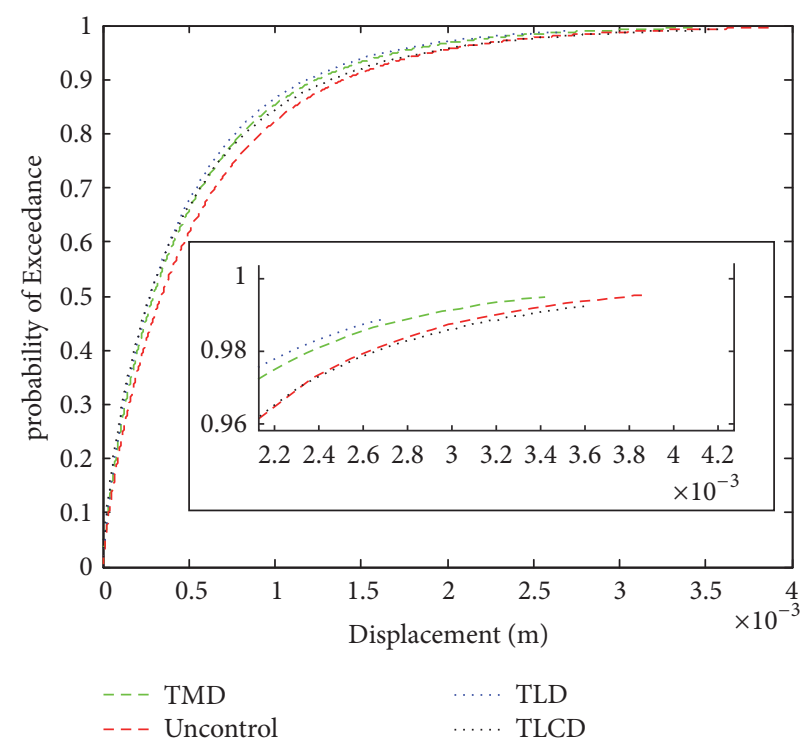

(b)

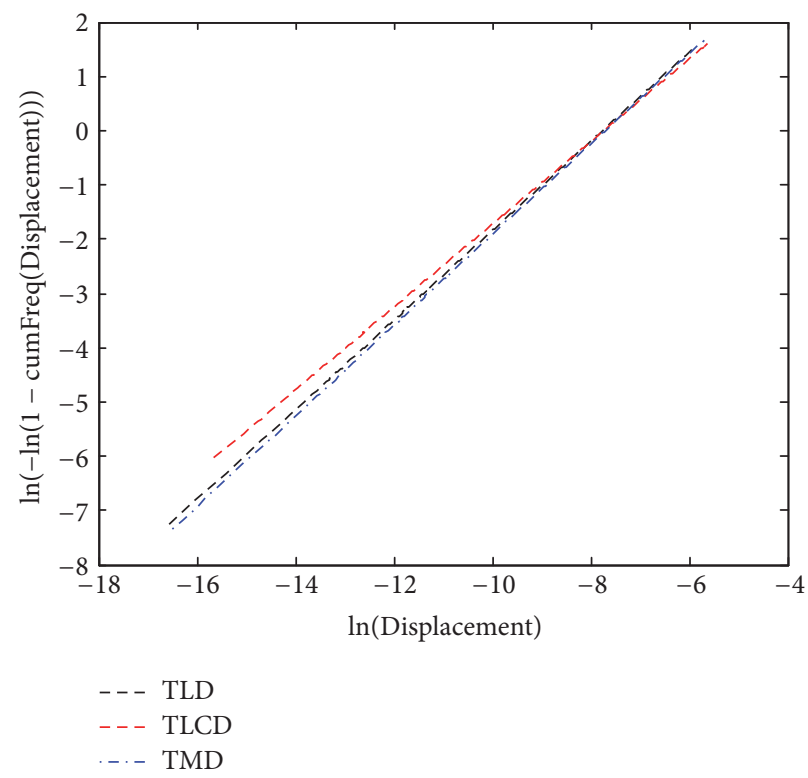

(c)

FIgURE 5: Weibull distribution for the controlling systems. (a) Linearized curve and fitted line comparison of TMD controller system. (b) Exceedance of the displacement probability. (c) Weibull slope.

and displacement response of the structure with the TLD controller are lower than the TMD and TLCD controllers. Based on the Kurtosis and Skewness results, the TLD probability distribution is better than TMD and TLCD. From Table 1, the statistical acceleration and displacement parameters for the TLD controlling system are more significant, but the TMD controlling system takes 7.85 seconds to control the structure response while the TLD takes 10.40 seconds.

The Weibull distribution of the structure's displacement of the uncontrolled, TMD, TLD, and TLCD systems is presented in Figure 5. The Weibull probability distribution is applied in this case for the absolute displacement response of each case. Figure 5(a) presents the TMD controller linearized displacement measurements for the Weibull parameters. The probability exceeded for the displacement is presented in Figure 5(b), and the Weibull parameter slope is presented in Figure 5(c). Also, the Weibull parameters for the controlling systems are presented in Table 2 .

The calculation of the autocorrelation coefficients for the fitting lines of the TMD, TLD, and TLCD is found 0.99, 0.97, and 0.95, respectively; the TMD fitting is presented in Figure 5(a). It means that the hypothesis testing for the TMD controller response with WD is high goodness-of-fit. In addition, the probability exceeded of the displacement shows that the TLD displacement is lower than TMD and TLCD. Also, the CDF for the TLD and TMD are very close. 


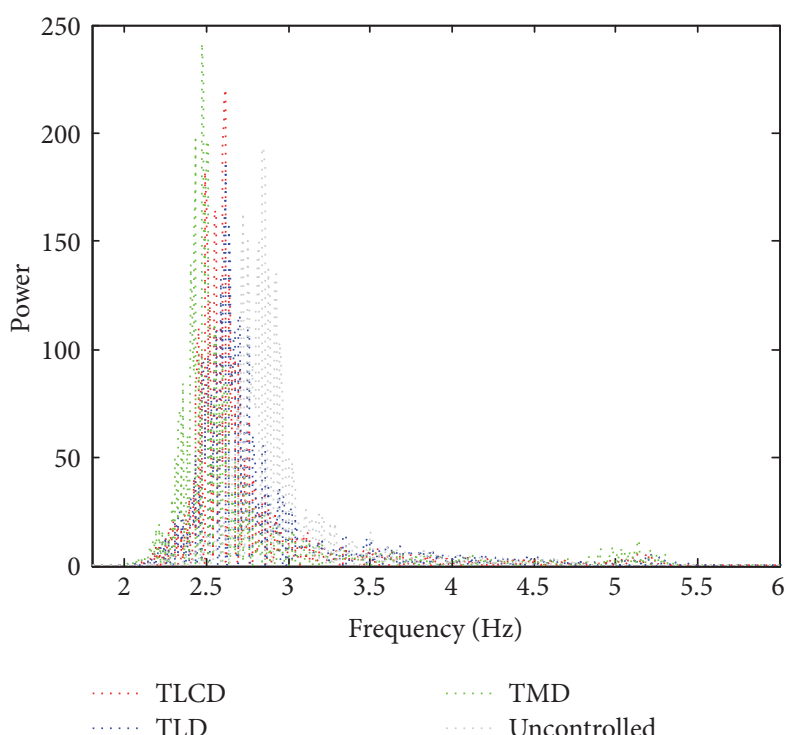

(a)

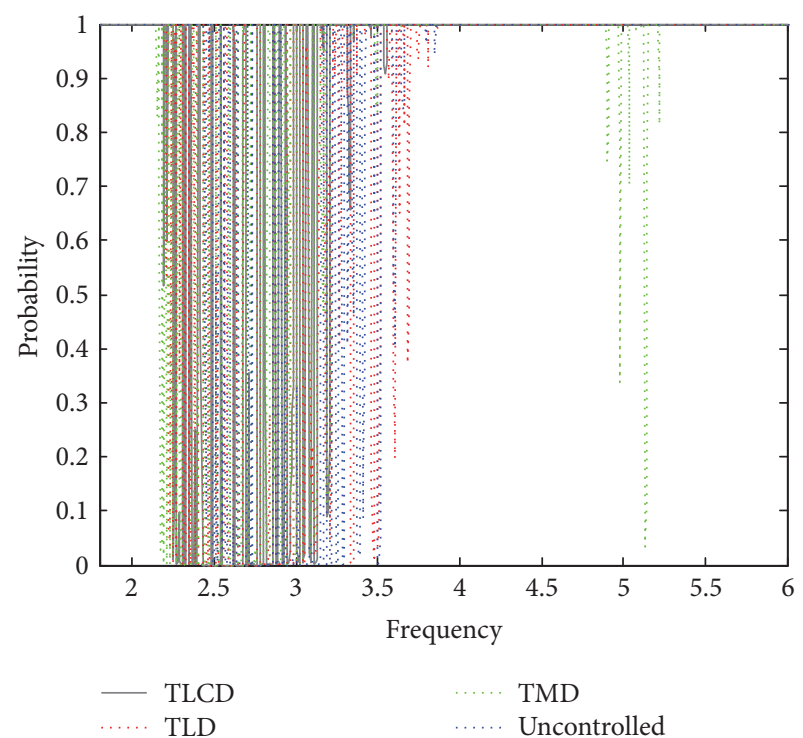

(b)

FIGURE 6: (a) Power spectrum density and (b) probability for the controlling systems.

TABLE 2: WD parameters for the uncontrolled and controlled displacement measurement.

\begin{tabular}{lcccc}
\hline WD parameters & Uncontrolled & TMD & TLD & TLCD \\
\hline$k$ & 0.85 & 0.80 & 0.82 & 0.84 \\
$c(m)$ & $5.24 e-4$ & $4.28 e-4$ & $4.32 e-4$ & $4.58 e-4$ \\
\hline
\end{tabular}

In addition, the Weibull slope presentation shows that the TMD slope is lower compared to TLD and TLCD of the displacement measurement. Also, the TMD and TLD Weibull slopes are very close. This means that the TMD controlling system is more reliable than other systems [35]. The two control systems TMD and TLD behaviors are close in a time domain performance. The WD parameters in Table 2 show that the scale parameter $(c)$ is very small and can be neglected, with close values for the three controlling systems. The shape parameter $(k)$ for the TMD is smaller compared to the TLD and TLCD. It means that the distribution of the structure performance with TMD controlling system is more normal than TLD and TLCD. The $k$ value measures the structure failure, and the smallest value of $k$ parameters refers to more stability and long life $[20,36,41]$. Therefore, the response of the TMD controlling system is more stable than other control systems.

The probabilities of the frequency contents are illustrated in Figure 6. The Lomb-Scargle power spectrum and probability methods were used to estimate the frequencies contents and the significant frequency components.

From Figure 6, the three frequency modes of the TMD and TLD controlling systems are 2.2, 2.5, and 5.2 and 2.2, 2.6, and $5.2 \mathrm{~Hz}$, respectively. Two frequency modes are shown only with the TLCD controlling system, 2.6 and $5.2 \mathrm{~Hz}$, whereas, with uncontrolled system, one frequency mode is shown at $2.8 \mathrm{~Hz}$ only. The probability exceeded for the frequency contents is between the values of 2.5

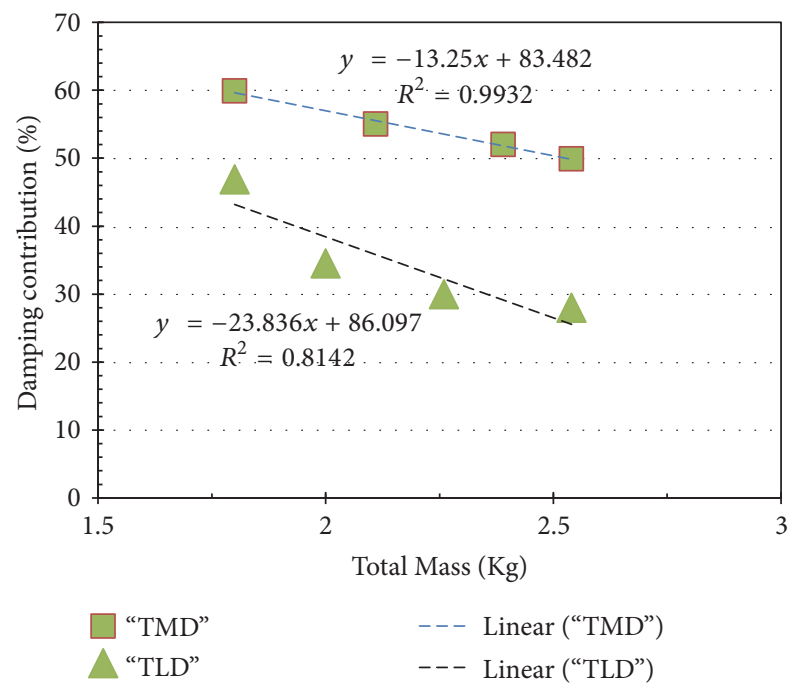

FIgURE 7: Damping contribution with effective mass of the TMD and TLD controlling systems.

and 3.5; 2.3 and 3.0; 2.3 and 3.1; and 2.1 and 3.0 for the uncontrolled, TLD, TLCD, and TMD systems, respectively. These results show that the damping contribution of the TMD and TLD controlling systems is more effective in suppressing the structure displacement. Therefore, the relation between mass effectiveness and damping contribution is presented in Figure 7. The effective masses of $1.8,2.1,2.39$, and $2.54 \mathrm{~kg}$ 


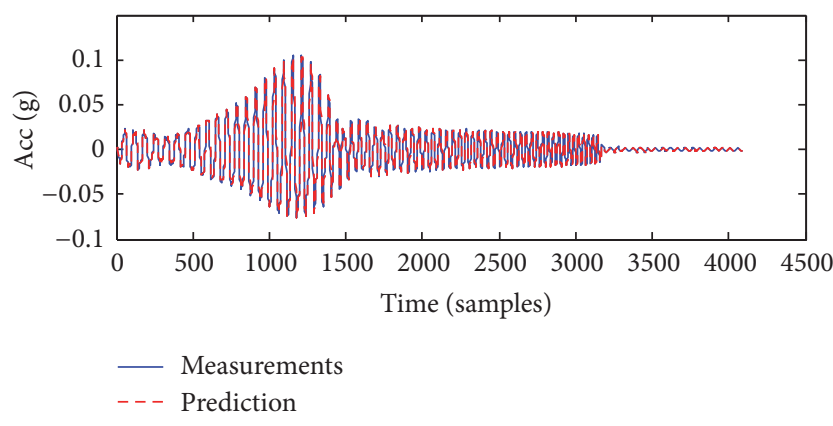

(a)

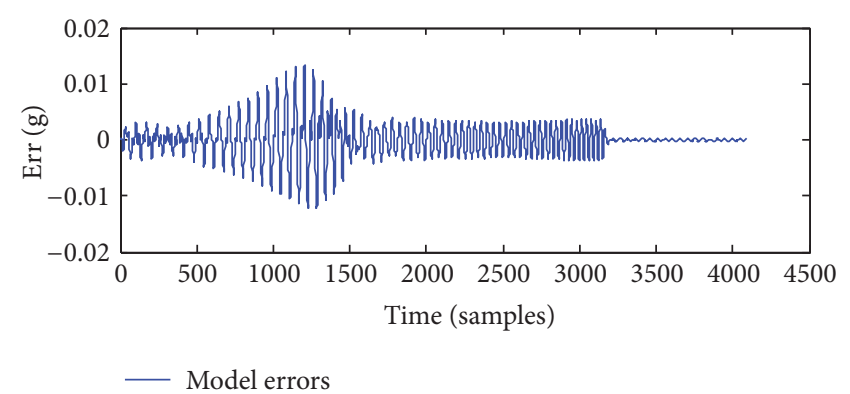

(b)

FIgURE 8: Prediction of actual response of the top floor of the structure for the (a) ARMANN models and (b) model errors.

TABLE 3: Statistical parameters analysis for the models' design.

\begin{tabular}{|c|c|c|c|c|c|}
\hline $\mathrm{AR}\left[\begin{array}{lll}n a & n b & n k\end{array}\right]$ & $E(\mathrm{~g})$ & $M(\mathrm{~g})$ & ARMA[ $\left.n a \begin{array}{llll}n b & n k & n c\end{array}\right]$ & $E(\mathrm{~g})$ & $M(\mathrm{~g})$ \\
\hline $\mathrm{AR}[111]$ & $2.27 e-4$ & $1.46 e-4$ & ARMA[1 1111$]$ & 0.0015 & 0.0010 \\
\hline $\mathrm{AR}\left[\begin{array}{lll}1 & 1 & 0\end{array}\right]$ & $2.05 e-4$ & $1.32 e-4$ & ARMA[1 1101$]$ & $1.46 e-4$ & $1.21 e-4$ \\
\hline $\mathrm{AR}\left[\begin{array}{lll}2 & 2 & 1\end{array}\right]$ & 0.0061 & 0.0041 & ARMA[ $\left.\begin{array}{llll}2 & 2 & 2 & 1\end{array}\right]$ & 0.0061 & 0.0041 \\
\hline $\mathrm{AR}\left[\begin{array}{lll}2 & 3 & 1\end{array}\right]$ & 0.0091 & 0.0061 & ARMA[1 2222$]$ & 0.0091 & 0.0061 \\
\hline $\mathrm{AR}\left[\begin{array}{lll}3 & 2 & 1\end{array}\right]$ & 0.0091 & 0.0061 & ARMA[ $\left[\begin{array}{llll}2 & 3 & 0 & 1\end{array}\right]$ & 0.0091 & 0.0061 \\
\hline $\operatorname{AR}\left[\begin{array}{lll}2 & 2 & 2\end{array}\right]$ & 0.0091 & 0.0061 & ARMA[[ $\left.\begin{array}{llll}3 & 2 & 0 & 1\end{array}\right]$ & 0.0091 & 0.0061 \\
\hline
\end{tabular}

and $1.8,2.0,2.26$, and $2.54 \mathrm{~kg}$ are utilized for the TMD and TLD, respectively; in addition, the damping contribution is calculated based on the power spectrum calculation by fast Fourier transformation method for each effective mass of the controlling system [9].

From Figure 7, the linear trend fitting for the TMD controlling system is linear with $R^{2}=0.99$, while the trend of the TLD fitting shows linear fitting with $R^{2}=0.81$. This can occur because the behavior of lamped mass TMD is more accurate because of solid mass movement while for TLD the liquid moving inside to provide damping shows more complicated behavior and energy dissipation process. The linear fitting of the two systems is considered as a damping index to predict the performance of the controlling system before actual applications. The TMD controlling system has high damping contribution than the TLD and TLCD. Accordingly, it is concluded that the TMD controlling system is more effective in the frequency and time domains than other controlling systems.

3.2. Response Model Identification Analysis. Based on the previous analysis, the structure design prediction model with the TMD controlling system is considered in this section. Two identification models, AR and ARMA, are evaluated using ANN. From (4), the AR model contains input parameters only without model error time delay. The autoregressive neural network (ARNN) model can be defined by the number of input parameters [na $n b n k]$, while ARMANN model can be defined by [na $n b n k n c]$. The acceleration of the input loads and third-floor response measurements are considered as input parameters. The third-floor response acceleration is utilized as an output parameter. Training the feedforward neural network (like AR model) and recurrent (like ARMA model) neural network is accomplished through iterative adjustments of the free parameters, that is, the weights and bias, of the network till we obtain the optimal values. There exist various learning algorithms, which are fundamental to the design of neural networks. The Levenberg-Marquardt learning algorithm is the most widely used algorithm for feedforward neural networks and recurrent neural network, which is used in this research $[28,43]$. Table 3 represents the statistical analysis for six trails of the model design for the AR and ARMA models with 100 iterations and 5 hidden neurons.

The trail models' performance evaluation show that two design models can be used for the identification of structures' behavior equipped with passive TMD controlling systems. The $E$ and $M$ values are shown equally from trails three to six for the AR and ARMA models. The obtained statistical analysis shows that the AR[110] and ARMA[1101] are more suitable to use in the current study, while it can be seen that the MAE and RMSE values for the two models are smaller than other models. The time delay is a main factor for the performance of the model design, where models' performance depends on the seismic inputs variables. The comparison between the AR and ARMA models shows that the ARMA model is better than AR model to predict the structure behavior. The ARMANN[$\left[\begin{array}{llll}1 & 1 & 0 & 1\end{array}\right]$ model can be used to detect the behavior of the controlled structure. In addition, the results show that the recurrent identification models are more effective than the feed forward to predict the behavior of the controlled structures. Figure 8 represents the measurements,

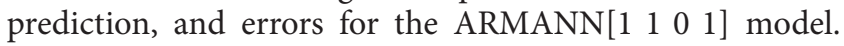
From this figure, it can be seen that the maximum error for the model is $0.013 \mathrm{~g}$, and the correlation coefficient between 
the measurements and prediction signals is high (0.98). This means that the ARMANN model is suitable for use as a tool to predict the behavior of controlled structures under dynamic loads.

\section{Conclusions}

Structural vibration control is a challenging and important problem in the structural engineering. The passive and active controlling systems are being used for the vibration control of buildings under various seismic excitations. The time series, frequency contents, and statistical analyses were discussed for TMD, TLD, and TLCD coupled with building structure systems in terms of vibration mitigation. A novel application for the probabilistic evaluation is utilized to analyze the behavior of structures coupled with controlling systems. The results show that the probability analysis of the performance of the TMD and TLD controlling systems is seen closed in time and frequency domains. In addition, the vibration damping of the TMD controlling system is highest than TLD and TLCD. Therefore, the TMD control system is more reliable controller than the TLD and TLCD systems.

Two autoregressive models (i.e., AR and ARMA) are used with neural networks solution to predict the behavior of structure coupled with TMD controlling system. The performances of two models are evaluated and the results showed that the ARMA model is more effective. The recurrent identifying models predict the behavior of the controlled structures in more accurate way. Evaluating the performance of the ARMANN model through experimental measurements showed that the model is a promising tool to predict the behavior of controlled structures under dynamic loads.

\section{Conflicts of Interest}

The authors declare that there are no conflicts of interest regarding the publication of this paper.

\section{Acknowledgments}

This research was supported by Basic Science Research Program through the National Research Foundation of Korea (NRF) funded by the Ministry of Science, ICT \& Future Planning (2017R1A2B2010120).

\section{References}

[1] I. Buckle, "Passive control of structures for seismic loads," in Proceeding of the 12th World Conference on Earthquake Engineering, p. 2825, Auckland, New Zeland, 2000.

[2] A. Arzeytoon, A. A. Golafshani, V. Toufigh, and H. Mohammadi, "Seismic performance of ribbed bracing system in passive control of structures," Journal of Vibration and Control, 2015.

[3] Y. Bigdeli and D. Kim, "Damping effects of the passive control devices on structural vibration control: TMD, TLC and TLCD for varying total masses," KSCE Journal of Civil Engineering, vol. 20, no. 1, pp. 301-308, 2015.
[4] H.-Y. Wang and J.-S. Chen, "Study of thin film transition liquid crystal display (TFT-LCD) optical waste glass applied in earlyhigh-strength controlled low strength materials," Computers and Concrete, vol. 5, no. 5, pp. 491-501, 2008.

[5] J. D. Marshall and F. A. Charney, "A hybrid passive control device for steel structures, I: Development and analysis," Journal of Constructional Steel Research, vol. 66, no. 10, pp. 1278-1286, 2010.

[6] J. D. Marshall and F. A. Charney, "Seismic response of steel frame structures with hybrid passive control systems," Earthquake Engineering and Structural Dynamics, vol. 41, no. 4, pp. 715-733, 2012.

[7] H. Adeli and H. Kim, "Recent advances and novel concepts for motion control of bridges and high-rise buildings under extreme winds and earthquakes," Asian Journal of Civil Engineering (building and housing), vol. 7, no. 4, pp. 335-342, 2006.

[8] F. Sakai, S. Takaeda, and T. Tamaki, "Tuned liquid column damper (TLCD) for cable-stayedbridges," in Proceeding of the specialty Conf. Invitation in Cable-stayed Bridges, Fukuoka, Japan.

[9] Y. Bigdeli, Development of Neuro-Controller, and TLCD-Structure coupled systems for Active Control of Irregular Structures [Ph.D. thesis], Kunsan National University, Kunsan, South Korea, 2014.

[10] Y. Chen, T. Cao, L. Ma, and C. Luo, "Structural vibration passive control and economic analysis of a high-rise building in Beijing," Earthquake Engineering and Engineering Vibration, vol. 8, pp. 561-568, 2009.

[11] K. Kasai, M. Nakai, Y. Nakamura, H. Asai, Y. Suzuki, and M. Ishii, "Current status of building passive control in Japan," in Proceeding of the 14th World Conference on Earthquake Engineering, Beijing, China, 2008.

[12] Y. Bigdeli and D. Kim, "Active control of 3-D irregular building by using energy based neuro-controller," Advances in Structural Engineering, vol. 17, no. 6, pp. 837-849, 2014.

[13] Y. Bigdeli and D. Kim, "Response control of irregular structures using structure-TLCD coupled system under seismic excitations," KSCE Journal of Civil Engineering, vol. 19, no. 3, pp. 672681, 2015

[14] J. N. Yang, A. Danielians, and S. C. Liu, "Hybrid control of nonlinear and hysteretic systems," J. of Engineering Mechanics, vol. 18, no. 1423-1439, pp. 1441-1457, 1992.

[15] S. Arangio and F. Bontempi, "Structural health monitoring of a cable-stayed bridge with Bayesian neural networks," Structure and Infrastructure Engineering, vol. 11, no. 4, pp. 575-587, 2014.

[16] A. Indriyatmoko, T. Kang, Y. J. Lee, and J. R. Kim, "ARMA model for neural networks predictor of DGPS carrier phase correction," Journal of the Korean Physical Society, vol. 51, pp. S62-S66, 2007.

[17] M. R. Kaloop and J. W. Hu, "Stayed-cable bridge damage detection and localization based on accelerometer health monitoring measurements," Shock and Vibration, vol. 2015, Article ID 102680, 11 pages, 2015.

[18] M. Kaloop and D. Kim, "GPS-structural health monitoring of a long span bridge using neural network adaptive filter," Survey Review, vol. 46, no. 334, pp. 7-14, 2014.

[19] V. Meruane and J. Mahu, "Real-time structural damage assessment using artificial neural networks and antiresonant frequencies," Shock and Vibration, vol. 2014, Article ID 653279, 14 pages, 2014. 
[20] M. R. Kaloop, J. W. Hu, and M. A. Sayed, "Yonjung HighSpeed Railway Bridge Assessment Using Output-Only Structural Health Monitoring Measurements under Train Speed Changing," Journal of Sensors, vol. 2016, Article ID 4869638, 15 pages, 2016.

[21] G. Deierlein, "Overview of a comprehensive framework for earthquake performance assessment," in Proceedings of the Performance-Based Seismic Design Concepts and Implementation, Bled, Slovenia, June-July 2004.

[22] B. Kim, "Probabilistic performance risk evaluation of infrastructure projects," in proceedings of the First International Symposium on Uncertainty Modeling and Analysis and Management (ICVRAM '11) and Fifth International Symposium on Uncertainty Modeling and Anaylsis (ISUMA), Hyattsville, MD, USA, April 2011.

[23] A. Cornell, "Hazard, ground motions and probabilistic assessments for PBSD," in Proceedings of the Performance-based seismic design concepts and implementation, vol. 1, Bled, Slovenia, June- July 2004.

[24] S. K. Verma, S. S. Bhadauria, and S. Akhtar, "Probabilistic Evaluation of Service Life for Reinforced Concrete Structures," Chinese Journal of Engineering, vol. 2014, Article ID 648438, 8 pages, 2014.

[25] J. Kralik, "A RSM method for nonlinear probabilistic analysis of the reinforced concrete structure failure of a nuclear power plant-type VVER 440," Engineering MECHANICS, vol. 18, no. 1, pp. 3-21, 2011.

[26] J. Matos, P. Cruz, I. Valente, L. Neves, and V. Moreira, "An innovative framework for probabilistic-based structural assessment with an application to existing reinforced concrete structures," Engineering Structures, vol. 111, pp. 552-564, 2016.

[27] M. Khashei and M. Bijari, "An artificial neural network (p, d, q) model for timeseries forecasting," Expert Systems with Applications, vol. 37, no. 1, pp. 479-489, 2010.

[28] M. El-Diasty, "An accurate heave signal prediction using artificial neural network," International Journal of Multidisciplinary and Current Research, vol. 2, pp. 989-993, 2014.

[29] A. Alarifi, N. Alarifi, and S. Al-Humidan, "Earthquakes magnitude predication using artificial neural network in northern Red Sea area," Journal of King Saud University-Science, vol. 24, no. 4, pp. 301-313, 2012.

[30] Y. Li, F. Zhang, B. Wu, and Q. Yang, "Simulation of structural seismic responses based on neural networks," in Proceedings of the 13th World Conference on Earthquake Engineering, p. 1534, Vancouver, British Columbia, Canada, August 2004.

[31] Y. Bigdeli and D. Kim, "Investigation of the performance of two passive controllers in mitigating the rotational response of irregular buildings," Advances in Materials Science and Engineering, vol. 2016, Article ID 1898792, 9 pages, 2016.

[32] S. M. Takhirov, G. L. Fenves, E. Fujisaki, and D. Clyde, "Ground motions for earthquake simulator qualification of electrical substation equipment," Peer report 2004/7, 2005.

[33] M. Kaloop, J. Hu, and Y. Bigdeli, "Identification of the response of a controlled building structure subjected to seismic load by using nonlinear system models," Applied Sciences, vol. 6, no. 10, p. 301, 2016.

[34] J. V. Seguro and T. W. Lambert, "Modern estimation of the parameters of the Weibull wind speed distribution for wind energy analysis," Journal of Wind Engineering \& Industrial Aerodynamics, vol. 85, no. 1, pp. 75-84, 2000.

[35] E. V. Zaretsky, R. C. Hendricks, and S. Soditus, "WeibullBased Design Methodology for Rotating Structures in Aircraft
Engines," International Journal of Rotating Machinery, vol. 9, pp. 313-325, 2003.

[36] http://www.weibull.com/hotwire/issue14/relbasics14.htm.

[37] J. Margetson and J. Laurillard, "Reliability analysis of structures with weibull distributions of load and strength to a given confidence level," Tech. Rep. Technical Report No. 182, Defense technical information center, USA, 1980.

[38] Vulpe A., Poteraşu V. F., and Cărăuşu A., "Failure probability and parameter estimation for structures with weibulldistributed strength," in Probabilistic Methods in the Mechanics of Solids and Structures. International Union of Theoretical and Applied Mechanics, S. Eggwertz and C. Lind, Eds., Springer, Heidelberg, Berlin, Germany, 1985.

[39] A. Schwarzenberg-Czerny, "The distribution of empirical periodograms: lomb-scargle and PDM spectra," Monthly Notices of the Royal Astronomical Society, vol. 301, no. 3, pp. 831-840, 1998.

[40] A. K. Azad, M. G. Rasul, R. Islam, and I. R. Shishir, "Analysis of wind energy prospect for power generation by three weibull distribution methods," in Proceedings of the 7th International Conference on Applied Energy (ICAE '15), pp. 722-727, March 2015.

[41] S. Hernandez, "Statistical analysis of weigh-in motion data for bridge design in vermont," Transportation Research Record: Journal of the Transportation Research Board TRC Report 14014, University of Vermont Transportation Research Center, 2014.

[42] M. H. Trauth, MATLAB ${ }^{\circledR}$ Recipes for Earth Sciences, 3rd edition, 2010.

[43] M. Norgaard, "Neural network based system identification toolbox, version 2," Tech. Rep. Tech. Report. 00-E-891, Department of Automation, Technical Un. of Denmark, 2000.

[44] J. Bodeux and J. Golinval, "Application of ARMAV models to the identification and damage detection of mechanical and civil engineering structures," Smart Materials and Structures, vol. 10, pp. 479-489, 2001.

[45] G. Atanasiu and V. Horga, "Grey-box direct identification of structures based on continous-time models," in Life-Cycle of Structural Systeme-Furuts, Frangopol And Akiyama (Eds), pp. 117-124, Taylor and Francis, London, 2015. 


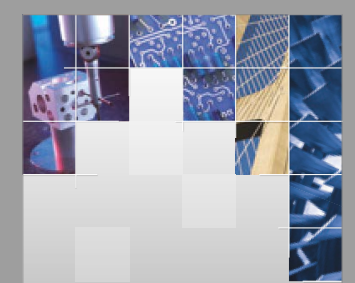

\section{Enfincering}
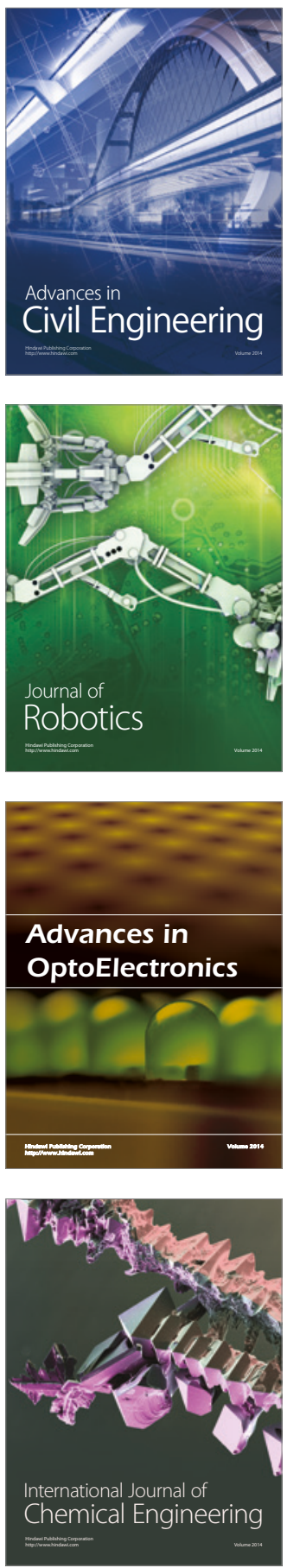

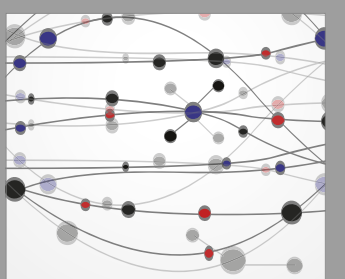

The Scientific World Journal

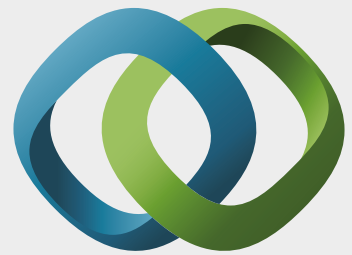

\section{Hindawi}

Submit your manuscripts at

https://www.hindawi.com
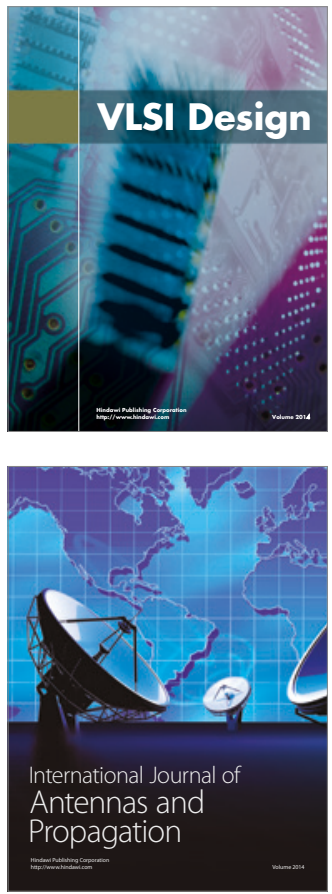

\section{Rotating}

Machinery
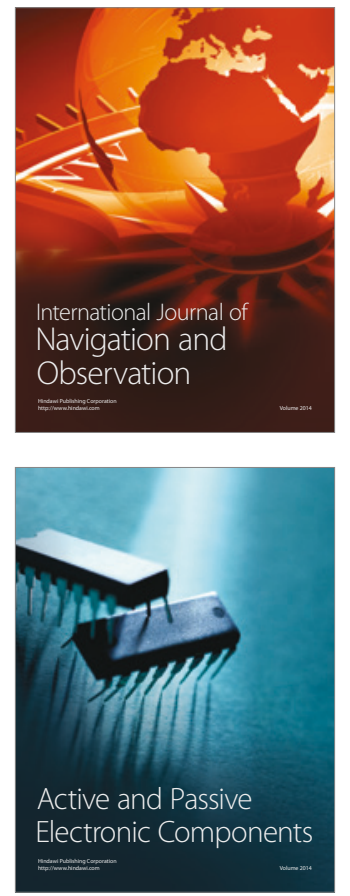
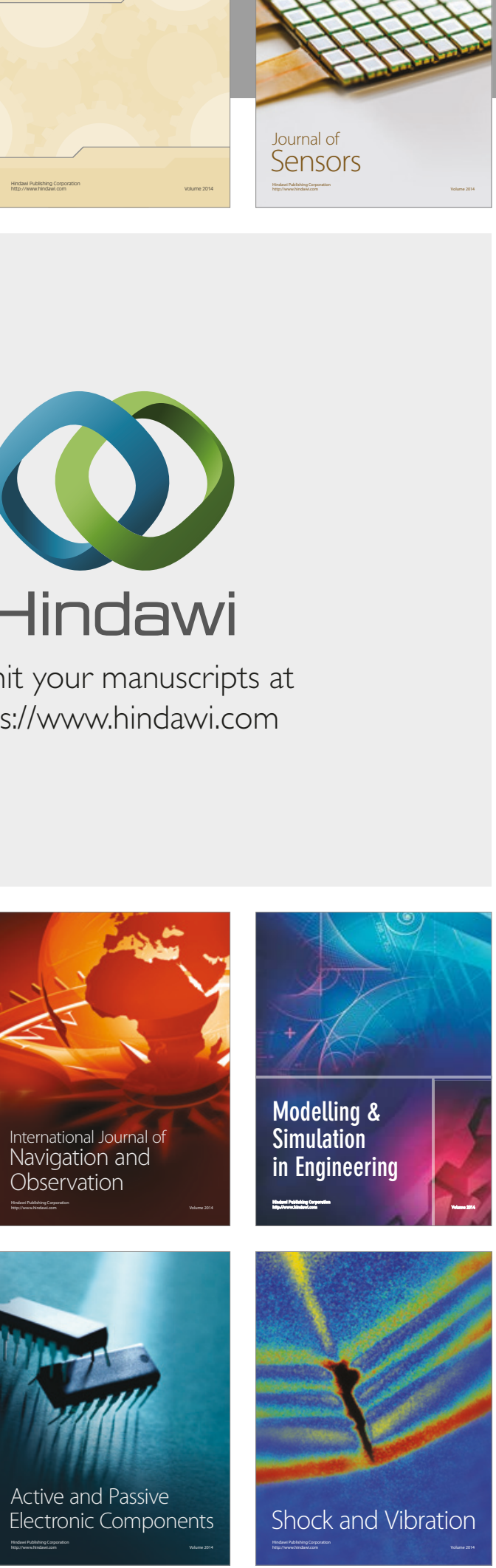
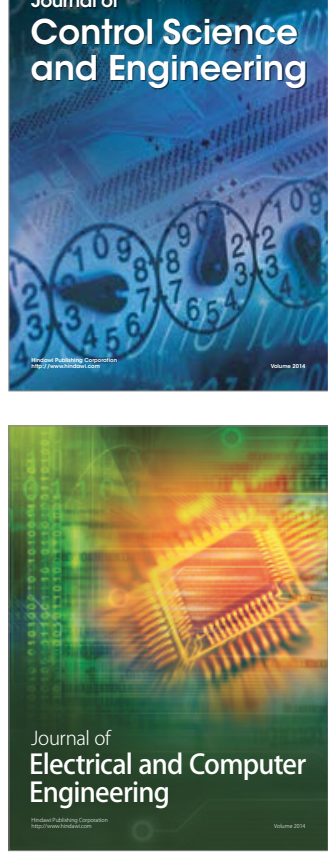

Distributed

Journal of

Control Science

and Engineering
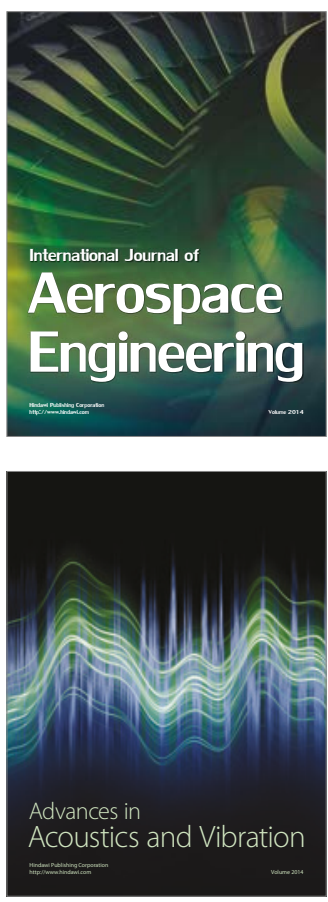

Sensor Networks 